\title{
Análisis cuantitativo en la influencia de la geomorfología en la estabilidad de taludes*
}

\author{
Sebastián Camacho Orozco ${ }^{* *}$ \\ Alfonso Mariano Ramos Cañón ${ }^{* * *}$ \\ Jorge Alberto Escobar Vargas ${ }^{* * * *}$ \\ Andrés Francisco Garzón González ${ }^{* * * *}$
}

Recibido: 01/12/2015 - Aceptado: 30/06/2016

DOI: 10.22395/rium.v16n30a1

\begin{abstract}
Resumen
La ocurrencia de deslizamientos está relacionada con unos factores que condicionan y otros que detonan los procesos de remoción en masa. Este trabajo contribuye a cuantificar la influencia de la geomorfología en la estabilidad de taludes como factor condicionante por medio de un análisis basado en el método de elementos finitos con un modelo constitutivo elastoplástico. Los análisis se realizaron a través de la simulación numérica de 9 modelos de taludes en tres dimensiones. Se obtuvo la variación espacial del tensor de esfuerzos y sus componentes por medio de las invariantes donde se refleja la influencia de la geoforma y el régimen de flujo en la estabilidad. Finalmente se calcula un indicador de falla potencial que cuantifica de manera unificada la influencia del esfuerzo de confinamiento y los esfuerzos de corte en el talud para determinar zonas de mayor susceptibilidad a la falla.
\end{abstract}

Palabras clave: geomorfología, taludes, elementos finitos, elastoplasticidad, falla.

* Artículo derivado de la investigación Análisis cuantitativo en la influencia de la geomorfología en la estabilidad de taludes, financiada por la Pontificia Universidad Javeriana entre enero 2013 y julio 2014. Instituciones participantes: Pontificia Universidad Javeriana, Bogotá, Colombia.

** Ingeniero civil. Pontificia Universidad Javeriana, Bogotá. Carrera 7 No. 40-62. Tel.: 3208320. Dirección electrónica: sebastian.camacho@javeriana.edu.co.

*** Profesor asociado. Instituto Geofísico Pontificia Universidad Javeriana, Bogotá. Carrera 7 No. 42-27. Tel.: 3208320. Ext. 6545. Dirección electrónica: a-ramos@javeriana.edu.co.

***** Profesor asistente. Departamento Ingeniería Civil. Pontificia Universidad Javeriana, Bogotá. Carrera 7 No. 40-62. Tel.: 3208320. Ext. 2719. Dirección electrónica: jorge-escobar@javeriana.edu.co.

****** Ingeniero civil. Pontificia Universidad Javeriana, Bogotá. Carrera 7 No. 40-62. Tel.: 3208320. Dirección electrónica: agarzong@javeriana.edu.co 


\title{
Quantitative analysis on the geomorphology influence in slope stability
}

\begin{abstract}
Landslide occurrence is associated with some conditioning factors and triggering landslide processes. This research contributes to quantify the influence of geomorphology as a conditioning factor in slope stability, via analysis based on a finite element method with an elastoplastic constitutive model. The analysis performs through a numerical simulation with nine three-dimensional slope models. The spatial variation of the stress tensor and its components were obtained by the invariants that reflect the influence of the geoform and flow regime in slope stability. Finally, the authors calculated an index potential failure that quantifies, in a unified manner, the influence of the confinement pressure and shear stress in the slope to determine a susceptibilty failure area.
\end{abstract}

Key words: geomorphology, slope, finite element method, elastoplasticity, failure. 


\section{INTRODUCCIÓN}

Los continuos deslizamientos dejan importantes pérdidas económicas impidiendo el desarrollo de una región. En Colombia, las zonas más propensas a deslizamientos se encuentran en la cadena montañosa de la cordillera de los Andes debido a sus condiciones topográficas, geológicas e hidrológicas [1]. Los deslizamientos en Colombia han ocupado el 16\% de la superficie nacional [2] (19'137239 ha) registrando en los últimos 30 años cerca de 28.258 muertes y más de 2300 millones de dólares en pérdidas por causa de estos eventos [3]. Para poderlos mitigar, se debe partir de la interpretación y el conocimiento de los factores geológicos, geotécnicos, geomorfológicos e hidrogeológicos de la zona de estudio, con el fin de relacionarlos con las causas que contribuyen y detonan el movimiento, dando como resultado, entre otros, la identificación del mecanismo de falla asociado a unas consecuencias [4]. Uno de los factores más importantes en la generación de procesos de remoción de masas es la geomorfología. El entendimiento de la influencia de la geomorfología en la estabilidad de taludes se ha desarrollado por medio de análisis cualitativos y cuantitativos. En términos cualitativos, autores como $[5,6]$ han estudiado el comportamiento espacial de la respuesta hidrológica debido al efecto de la geoforma en taludes, por medio del análisis de nueve geoformas, resultado de la combinación de tres formas de perfil y en planta (recto, convexo, cóncavo).

Wysocki y Schoeneberger [6] describen un comportamiento divergente de las líneas de flujo en formas convexas y un comportamiento convergente en formas cóncavas que afecta directamente la estabilidad del talud.

En términos cuantitativos, Iverson y Ried [7] establecen indicadores potenciales de falla a través de simulaciones numéricas por el método de elementos finitos en taludes modelados en dos dimensiones influenciados por la geomorfología y las presiones de poros en el modelo. A pesar de los trabajos desarrollados, no se conoce una propuesta que muestre de forma espacial cuantitativa la influencia de las geoformas en la estabilidad de taludes. [5, 6, 7] muestran que el problema geométrico debe abordarse incluyendo las tres dimensiones espaciales, dado que las aproximaciones de deformación plana (dos dimensiones) esconden aspectos relevantes en la generación de esfuerzos de corte en ubicaciones determinadas en el talud.

Este trabajo aborda la deficiencia anteriormente mencionada a través de un análisis cuantitativo con base en simulación numérica con el método de elementos finitos de las nueve posibles geoformas que surgen de combinar la vista en perfil y en planta del tipo recto, cóncavo y convexo, también estudiadas de forma descriptiva por [5, 6]. Con base en los resultados se analiza la distribución espacial de las componentes de esfuerzo-deformación por corte e invariantes de esfuerzo en las nueve geoformas. 
Con base en lo anterior, se calcula un indicador de falla potencial que tiene la potencialidad de tener en cuenta los dos aspectos más influyentes en la estabilidad de taludes con un modelo cuya envolvente de falla esté en función del cortante máximo y el esfuerzo medio efectivo. El indicador de falla potencial indica qué tan lejos en función de esfuerzos se encuentra diferentes puntos materiales del talud para llegar a la falla. Lo anterior permite resaltar cuáles zonas deben ser intervenidas inicialmente y diferenciar cuáles geoformas tienen mayor propensión a la falla, dada su cercanía a la envolvente de resistencia.

\section{MODELO CONSTITUTIVO}

Para la realización de la modelación numérica, se seleccionó un modelo constitutivo elasto-plástico del tipo Mohr Coulomb que se encuentra implementado en el software Abaqus de elementos finitos. Los aspectos más importantes que incluye el modelo constitutivo son [8]:

- Función de fluencia: es gobernada por la ecuación (1) la cual está definida por el estado y parámetros del material a través de invariantes de esfuerzos.

$$
F=R m c \cdot q-p \cdot \tan \phi-c
$$

$F=$ función de fluencia

$R m c=$ es una medida en la forma de la superficie de fluencia en función del ángulo de fricción $(\phi)$ y el ángulo de Lode $(\Theta)$ (ecuación 2).

$$
R m c=\frac{1}{\sqrt{3 \cdot \cos \phi}} \cdot \sin \left(\Theta+\frac{\pi}{3}\right)+\frac{1}{3} \cos \left(\Theta+\frac{\pi}{3}\right) \cdot \tan \phi
$$

$p=$ primera invariante de esfuerzos.

$q=$ segunda invariante de esfuerzos.

$c=$ cohesión del material.

$\phi=$ ángulo de fricción interna.

- Función de potencial plástico: establece la magnitud y dirección de las deformaciones plásticas por medio de una regla de flujo no asociada (ecuación 3).

$$
G=\sqrt{(E \cdot c \cdot \tan \psi)^{2}+(R m w \cdot q)}-p \cdot \tan \psi
$$


$G=$ potencial plástico

$E=$ es la excentricidad meridional la cual controla la forma de la función $G$ en el plano meridional. El plano meridional representa un corte de la superficie del potencial plástico, donde la dirección de las deformaciones plásticas está perpendicular a la superficie de potencial plástico [8].

$\psi=$ ángulo de dilatancia.

$R m w$ = función elíptica propuesta por Menetrey y Willam [9] (ecuación 4) para que la forma de la superficie del potencial plástico en el modelo Mohr Coulomb tenga esquinas suaves en el espacio principal de esfuerzos. La suavización está en función de la excentricidad desviadora (e) (ecuación 5) y el ángulo de Lode $(\Theta)$.

$$
\begin{gathered}
R m w=\frac{4 \cdot\left(1-e^{2}\right) \cdot(\cos \Theta)^{2}+(2 e-1)^{2}}{2 \cdot\left(1-e^{2}\right) \cdot \cos \Theta+(2 e-1) \cdot \sqrt{4 \cdot\left(1-e^{2}\right) \cdot(\cos \Theta)^{2}+5 e^{2}-4 e}} \cdot R m c \\
e=\frac{3-\sin \phi}{3+\sin \phi}
\end{gathered}
$$

- Función de endurecimiento: el comportamiento de endurecimiento o ablandamiento del material depende de una cohesión equivalente para cada nivel de deformaciones plásticas. La evolución de la superficie de fluencia dada por el endurecimiento es del tipo isotrópico, y es controlado directamente por el usuario ingresando la cohesión equivalente para las deformaciones plásticas en el proceso de carga.

Los parámetros del modelo constitutivo elasto-plástico se definieron con base en valores típicos de arenas limosas propuestos por Puri y Bowles [10-11] (tabla 1).

Tabla 1. Valores típicos de parámetros para el modelo elastoplástico de Mohr Coulomb para arenas limosas

\begin{tabular}{|c|c|c|}
\hline \multicolumn{3}{|c|}{ Parámetros del modelo elasto plástico } \\
\hline Ángulo de fricción $\left(\varphi^{\circ}\right)$ & Ángulo de dilatancia $\left(\psi^{\circ}\right)$ & Cohesión $(\mathrm{kPa})$ \\
\hline 34 & 5 & 25 \\
\hline$\gamma\left(\mathrm{kN} / \mathrm{m}^{3}\right)$ & Módulo de elasticidad $(\mathrm{kPa})$ & Relación de poisson \\
\hline 19 & 18000 & 0.33 \\
\hline
\end{tabular}

Fuente: elaboración propia 


\section{MODELO SUCCIÓN HUMEDAD}

Para simular la presencia de nivel freático, en cada talud se define una zona saturada, abajo del nivel freático y una zona parcialmente saturada, arriba del nivel freático. La zona saturada es gobernada bajo la ley de Darcy donde se establecen dos alturas piezométricas para lograr un flujo estacionario a lo largo del talud. La zona parcialmente saturada es representada por la ecuación analítica propuesta por Van Genuchten [12].

En la figura 1 se observa las condiciones de frontera definidas en el modelo de succión humedad para la conformación de nivel freático y en la tabla 2 se presenta los parámetros seleccionados del modelo succión humedad.

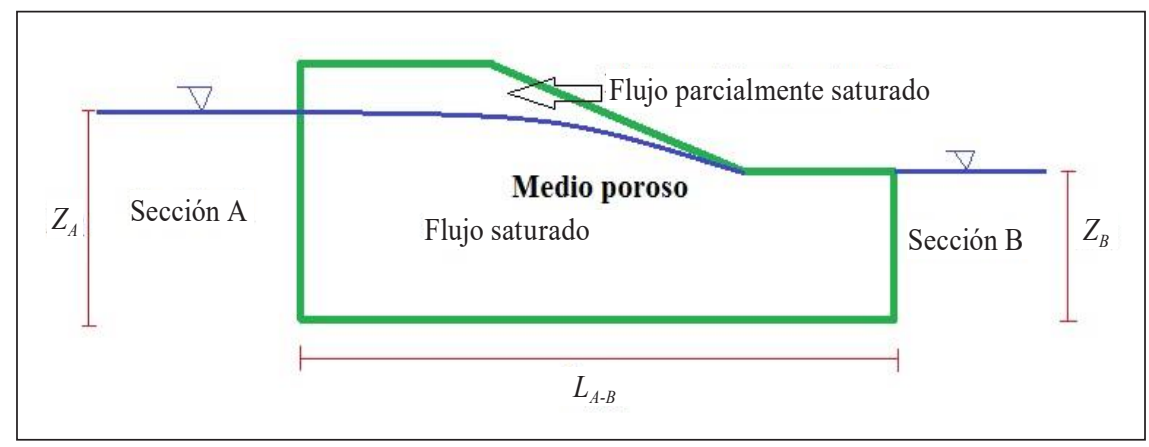

Figura 1. Condiciones de flujo en un medio poroso.

Fuente: elaboración propia

Tabla 2. Valores típicos de arenas limosas en el modelo succión humedad

\begin{tabular}{|c|c|c|c|}
\hline \multicolumn{4}{|c|}{ Parámetros del modelo succión humedad } \\
\hline$\omega(\%)$ & $e$ & $\operatorname{Sr}(\%)$ & $K(\mathrm{~m} / \mathrm{s})$ \\
\hline 20 & 0.85 & 62.8 & 0.0001 \\
\hline
\end{tabular}

Fuente: elaboración propia

$\omega=$ Contenido de humedad

$e=$ Relación de vacíos

$S r=$ Grado de saturación inicial

$K=$ Coeficiente de permeabilidad

Para establecer la condición de saturación parcial se conformó la curva de retención de humedad por medio de la ecuación analítica propuesta por Van Genuchten (ecuación 6) [12], que relaciona el grado de saturación y el esfuerzo de succión en el medio poroso. En la tabla 3 se definen los parámetros de la ecuación de Van Genu- 
chten [13]. La figura 2 muestra la relación succión-grado de saturación resultante de la aplicación del modelo mencionado.

$$
S r=\left[\frac{1}{1+(\rho \psi)^{n}}\right]^{m}
$$

$S r=$ grado de saturación

$\rho, m, n=$ parámetros dependientes de la gradación y clasificación textural del suelo propuestos por Van Genuchten

$\psi=$ esfuerzo de succión $(\mathrm{kPa})$

Tabla 3. Parámetros típicos de una arena limosa para la ecuación de Van Genuchten

\begin{tabular}{|c|c|c|}
\hline$\rho\left(m^{-1}\right)$ & $m$ & $n$ \\
\hline 3.1 & 1.27 & 0.213 \\
\hline \multicolumn{3}{|c|}{ Fuente: elaboración propia } \\
\end{tabular}

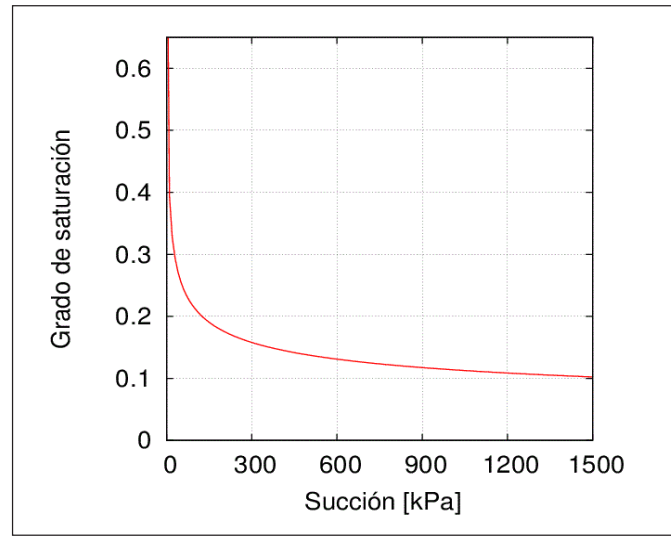

Figura 2. Relación succión-grado de saturación para arena limosa con el modelo de Van Genuchten.

Fuente: elaboración propia

\section{SIMULACIÓN NUMÉRICA EN TALUDES}

Con el objetivo de analizar el efecto de la geoforma en la estabilidad de taludes se simularon 18 modelos de taludes: nueve modelos en términos de esfuerzos totales y nueve en términos de esfuerzos efectivos. En la figura 3 se observan las 9 geoformas analizadas. Las geoformas son producto de combinar la disposición en planta y en perfil recto, cóncavo y convexo. 


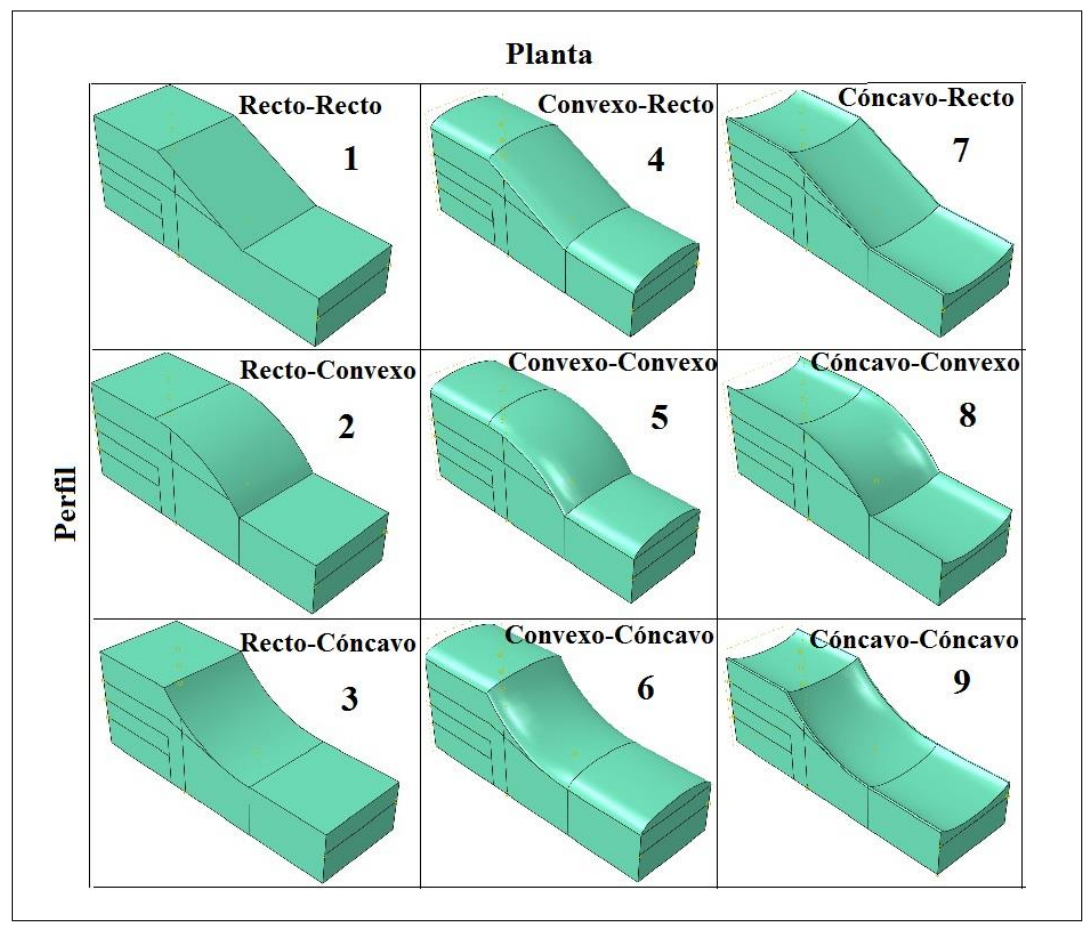

Figura 3. Combinaciones de geoformas en planta y perfil.

Fuente: elaboración propia

\subsection{Geometría}

Las distancias Fe y Fp (figura 4) se determinaron por medio de un análisis paramétrico mostrado en la figura 5 debido al efecto que genera la restricción de los desplazamientos en los nodos (dirección x) en la distribución de esfuerzos.

La asignación numérica de la geometría del talud se seleccionó cuidando que las condiciones de contorno de desplazamientos en los límites del modelo no afectaran los resultados en términos de esfuerzos de corte tanto en la corona como en la pata del talud.

Las figuras $5 a$ y $5 b$ muestran la variación de los esfuerzos de corte en una sección en la corona y en la pata del talud, respectivamente, en función de la altura $\mathrm{H}$ del talud (figura 4). La altura $\mathrm{H}$ del talud se fijó con valor de $25 \mathrm{~m}$. Se observa que para fronteras cercanas a la pata y a la corona del talud $(0.4 \mathrm{H}$ a $1.4 \mathrm{H})$ se presenta variación significativa de los esfuerzos de corte en profundidad. Para valores de distancia de la frontera izquierda a la corona de los taludes superiores a $\mathrm{Fc}=1.8 \mathrm{H}$ no se observa 
cambio representativo de los esfuerzos de corte (figura 5a). Comportamiento similar se presenta para la pata del talud con distancias mayores a la frontera derecha de $\mathrm{Fp}=1.6$ $\mathrm{H}$ (figura 5b).

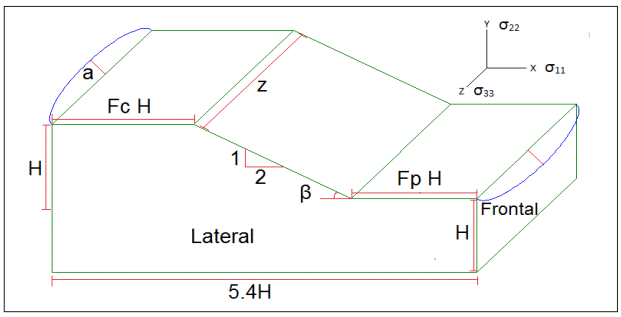

Figura 4. Parametrización de la geometría del talud.

Fuente: elaboración propia

Tabla 4. Dimensiones seleccionadas para la geometría del talud

\begin{tabular}{|c|c|c|c|c|c|}
\hline$H(m)$ & $a(m)$ & $z(m)$ & $\beta^{\circ}$ & $F c$ & $F p$ \\
\hline 25 & 6.75 & 45 & 26.56 & 1.8 & 1.6 \\
\hline
\end{tabular}

Fuente: elaboración propia
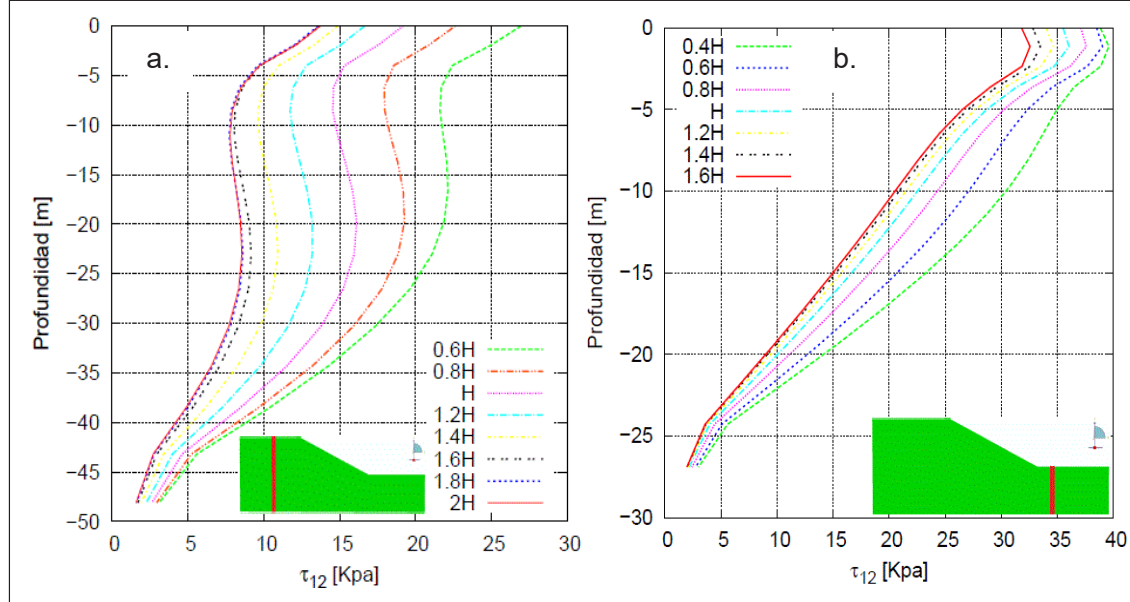

Figura 5. Variación de los esfuerzos de corte en sección de análisis:

a. Para diferentes distancias de la frontera izquierda del modelo con la corona del talud;

b. Para diferentes distancias de la frontera derecha del modelo con la pata del talud.

Fuente: elaboración propia

\subsection{Condiciones iniciales de carga y de desplazamiento para la simulación numérica}

El estado de esfuerzos inicial fue del tipo geoestático simulando la conformación del talud por etapas sucesivas de carga. En las condiciones de frontera se restringieron los desplazamientos en los nodos: en la componente $(\mathrm{X})$ para las caras frontales, en la 
componente (Y) para la base del talud, y en la componente $(\mathrm{Z})$ para las caras laterales. El sistema coordenado se observa en la figura 4. Para lograr la conformación del nivel freático en cada talud, se seleccionó un flujo estacionario donde se definieron dos alturas piezométricas de 35 y 15 metros en los costados frontales del plano (YZ) según la figura 4.

\subsection{Enmallado}

El diseño de la malla se basó en los criterios del factor de forma, angulosidad y relación de área de elementos tetraédricos propuestos en [14], logrando estabilidad en la interpolación de los resultados. El refinamiento de la malla se realizó en las zonas de mayor concentración de esfuerzos obteniendo un número aproximado de $10^{5}$ elementos en cada uno de los modelos. Debido a la cantidad significativa de elementos se seleccionó un método de solución tipo iterativo para disminuir el tiempo y memoria computacional requerida en cada simulación.

\section{ANÁLISIS DE RESULTADOS}

Se realizaron 2 tipos de resultados para analizar la variación espacial de esfuerzos por causa del factor geomorfológico.

- Distribución de los contornos de esfuerzos efectivos y grados de saturación

- Cálculo de indicadores potenciales de falla en términos de esfuerzos totales y efectivos

\subsection{Contornos de esfuerzos y grados de saturación}

En términos de esfuerzos efectivos se analizó la distribución de los esfuerzos y grados de saturación a lo largo de cada talud en una vista en planta. En la figura 6 se presentan los contornos de grados de saturación y el valor del máximo componente del tensor de esfuerzos para cada modelo. El número de cada modelo corresponde al mostrado en la figura 3. En los modelos rectos en planta (figura 6a) se presenta un comportamiento uniforme en el grado saturación de la pata del talud. El efecto de la geoforma en la condición espacial tridimensional se observa predominante en los modelos convexos y cóncavos. En los modelos convexos (figura 6b) se evidencia un comportamiento divergente de las líneas de flujo del centro de simetría del talud reflejado en saturación del $100 \%$ en la pata y extremos del mismo. Estas zonas tienen bajas profundidades por lo que las presiones de poros generadas por la dinámica del flujo hacen que se presenten esfuerzos efectivos negativos, encontrándose que la resistencia al corte en estas zonas es muy baja con relación a la parte central del talud. Situación contraria ocurre en modelos cóncavos en planta (figura 6c). En estos modelos se presenta convergencia 
de las líneas de flujo en el centro de la parte baja del talud generando saturaciones del $100 \%$, de forma tal que los esfuerzos efectivos son bajos en esta zona y se espera que la resistencia del talud esté modulada por la propagación de la falla a partir de esta zona.

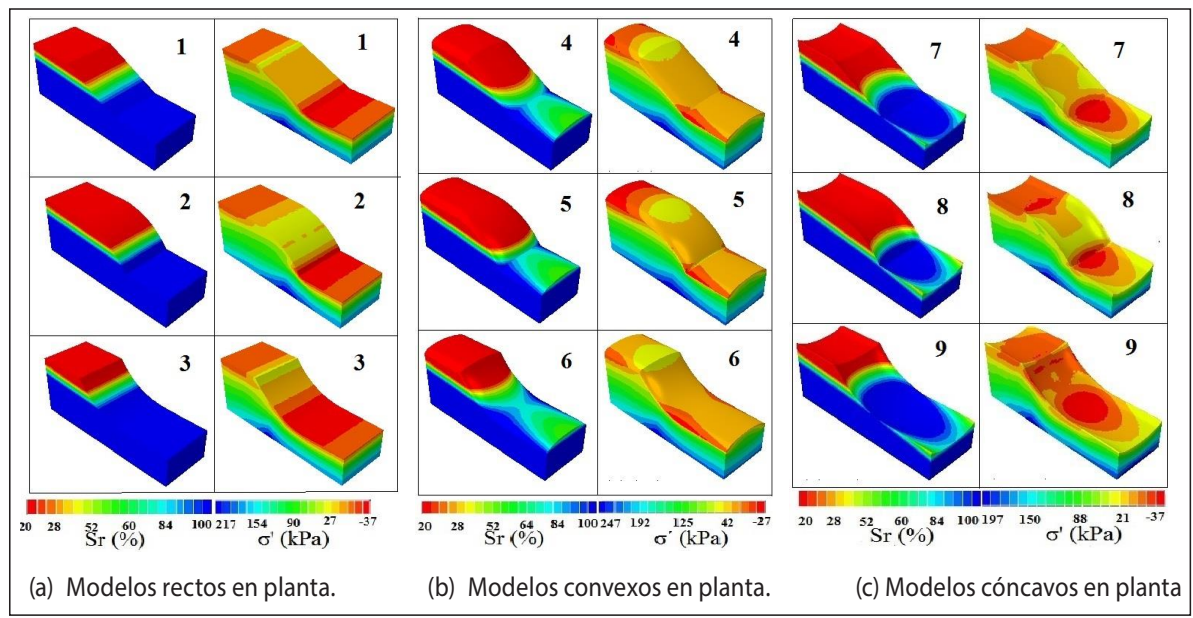

Figura 6. Contornos de grados de saturación y el máximo componente del tensor de esfuerzos efectivos.

Fuente: elaboración propia

Para poder analizar de mejor manera las diferencias en los esfuerzos que genera la condición tridimensional observadas en la figura 6 , se seleccionaron dos líneas de análisis (extremo y centro) (figura 7).

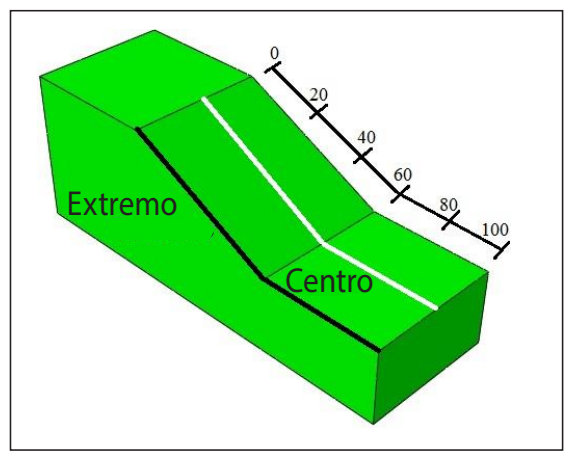

Figura 7. Localización de las líneas de análisis.

Fuente: elaboración propia

Según la figura 7, entre 40 y 100 metros corresponden aproximadamente a las zonas saturadas en cada uno de los modelos. En las zonas saturadas en la línea de análisis del centro, los modelos cóncavos (modelos 3, 7, 8, 9) en planta presentan esfuerzos efectivos de aproximadamente $-35 \mathrm{kPa}$, a diferencia de modelos convexos 
(modelos 4, 5, 6), que presentan mayores esfuerzos efectivos en las zonas parcialmente saturadas por causa de la divergencia del flujo. En la línea de análisis del extremo, los modelos convexos son más susceptibles a deslizarse, a diferencia de modelos cóncavos. Los modelos rectos presentan disminuciones en los esfuerzos en las dos líneas analizadas.

\subsection{Indicadores potenciales de falla}

Para poder cuantificar la influencia de las geoformas en la estabilidad de los taludes analizados, se adoptó la propuesta del indicador de falla $(\Phi)$ presentado por Iverson y Ried [7] (ecuación 7). Este indicador tiene la potencialidad de tener en cuenta, en términos de esfuerzos que la propensión a la falla de un talud utilizando en un modelo constitutivo con envolvente de falla del tipo Mohr Coulomb es proporcional al cortante máximo desarrollado en el cuerpo del mismo, e inversamente proporcional al esfuerzo medio efectivo de los puntos materiales en el talud.

$$
\Phi=\frac{\left|\tau_{\max }\right|}{-p}
$$

El indicador se explica de la siguiente manera: por un lado, en el denominador, cuando el esfuerzo medio efectivo de los puntos materiales es bajo, indica que la contribución del esfuerzo de confinamiento para desarrollar mayor resistencia al corte es baja. Por otro lado, si se están desarrollando esfuerzos de corte muy altos, quiere decir que estará muy cerca de la envolvente de falla del material. Se obtiene el valor absoluto del esfuerzo máximo de corte, dado que su signo solo indica la distorsión preponderante de los elementos materiales. Altos valores de $\Phi$ indican que la trayectoria de esfuerzos que necesita cada punto material para llegar a la falla es baja. Si el valor de $\Phi$ es pequeño indica que dichos puntos materiales pueden sostener mayores cargas adicionales antes que esté cerca a la envolvente de falla.

El indicador de falla $\Phi$ puede compararse con el parámetro relacionado con el ángulo de fricción máximo movilizado en el espacio de invariantes p-q para el caso axisimétrico representado por el valor de $M$ (ecuación 8).

$$
M=\frac{6 \cdot \sin \phi}{3-\sin \phi}=\frac{q}{p}
$$


Para el material seleccionado, el máximo valor que puede tener el parámetro $M$ es de 1.37 calculado con base en el ángulo de fricción pico del material (tabla 1). Se definió un rango de $\Phi$ entre 0.001 y 1.4.

Los indicadores de falla se evaluaron en cada uno de los modelos por medio de contornos para lograr observar zonas susceptibles a deslizamientos. Las zonas de análisis se definieron por medio de cortes transversales en el centro de cada uno de los taludes para que fueran representativos del talud. Se analizaron indicadores de falla en términos de esfuerzos totales y efectivos para observar la influencia del comportamiento divergente o convergente del flujo.

Los modelos convexos en perfil en la figura 8 (modelos 2,5 y 8 ) presentan indicadores cercanos o superiores a 1.0 en la pata del talud, a diferencia de modelos cóncavos (modelos 3, 6 y 9), los cuales presentan altos indicadores en la corona del talud. Es decir, la geoforma cóncava o convexa genera concentración de esfuerzos de corte debido a la no homogeneidad en perfil. Los modelos con geoformación en perfil presentan indicadores más bajos que los anteriores (modelos 1, 4 y 7). Lo anterior indica que para taludes sin presencia de agua, los modelos con geometría recta en perfil son más estables en comparación con modelos cóncavos y convexos. Si se espera que se genere una falla del tipo progresivo, en el talud con perfil convexo iniciará en la parte baja del talud y se propagará hacia arriba dado que los contornos de mayores valores se ubican inmediatamente arriba de los puntos de concentración del indicador $\Phi$. Por otro lado, en los taludes de perfil cóncavo, aunque hay concentración de valores altos del indicador $\Phi$ cerca de la corona del talud, es más difícil que se desarrolle una falla progresiva hacia la pata del talud, dado que hacia debajo de la corona los valores del indicador son más pequeños, haciendo más difícil la propagación de la superficie de discontinuidad. Ninguno de los taludes en estado seco alcanza un indicador mayor a 1.37 logrando una estabilidad en la zona analizada. Resultados similares encontraron Iverson y Ried [7], donde los modelos con geometría convexa y transición entre convexo y cóncavo son considerados más susceptibles a la falla.

Los indicadores potenciales de falla en taludes con presencia de nivel freático están condicionados por el comportamiento convergente y divergente del flujo. Los modelos con geometría cóncava (modelos 7, 8 y 9) y recta (1, 2 y 3 ) en perfil presentan altos indicadores en las zonas saturadas; en cambio, en modelos convexos (modelos 4, 5 y 6) son considerados menos susceptibles a fallar por causa de la divergencia del flujo observando bajos indicadores en zonas parcialmente saturadas (figura 9). 


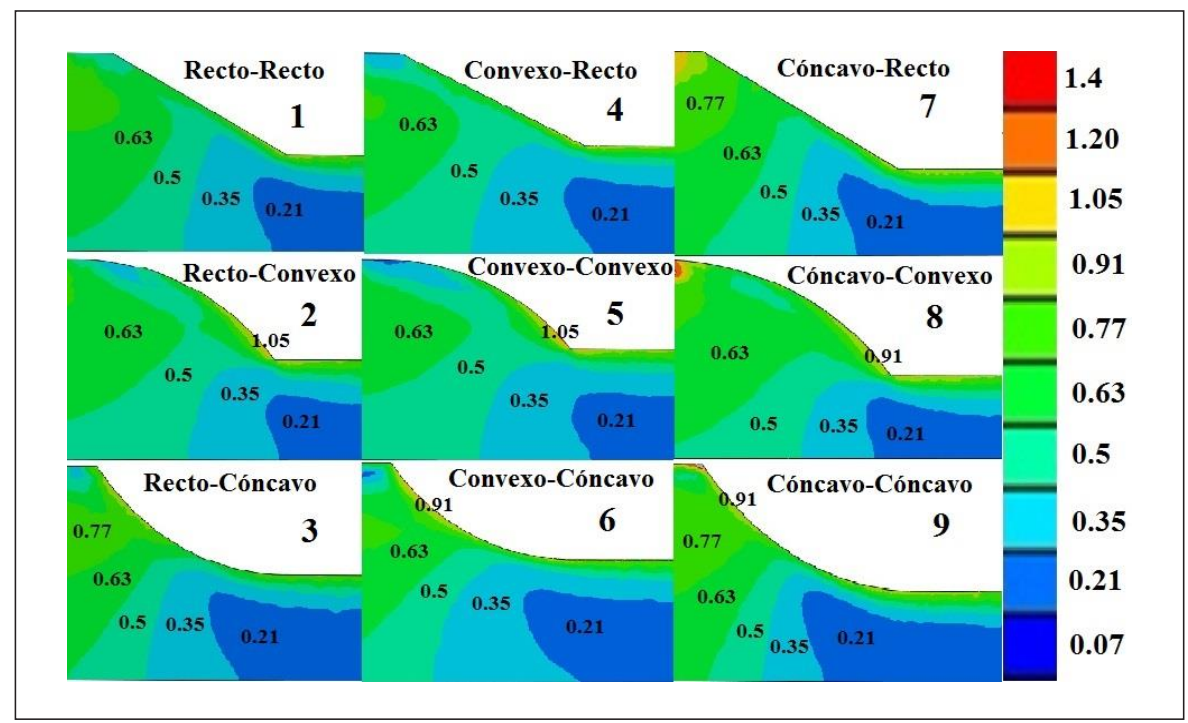

Figura 8. Contornos de indicadores potenciales de falla en taludes sin la presencia de agua. Fuente: elaboración propia

A diferencia de los modelos secos, los taludes con presencia de flujo presentan zonas inestables logrando alcanzar indicadores mayores a 1.37 en modelos rectos y cóncavos en planta. Como se espera, la inestabilidad potencial es acentuada en los taludes que presentan flujo en comparación con aquellos que no tienen flujo.

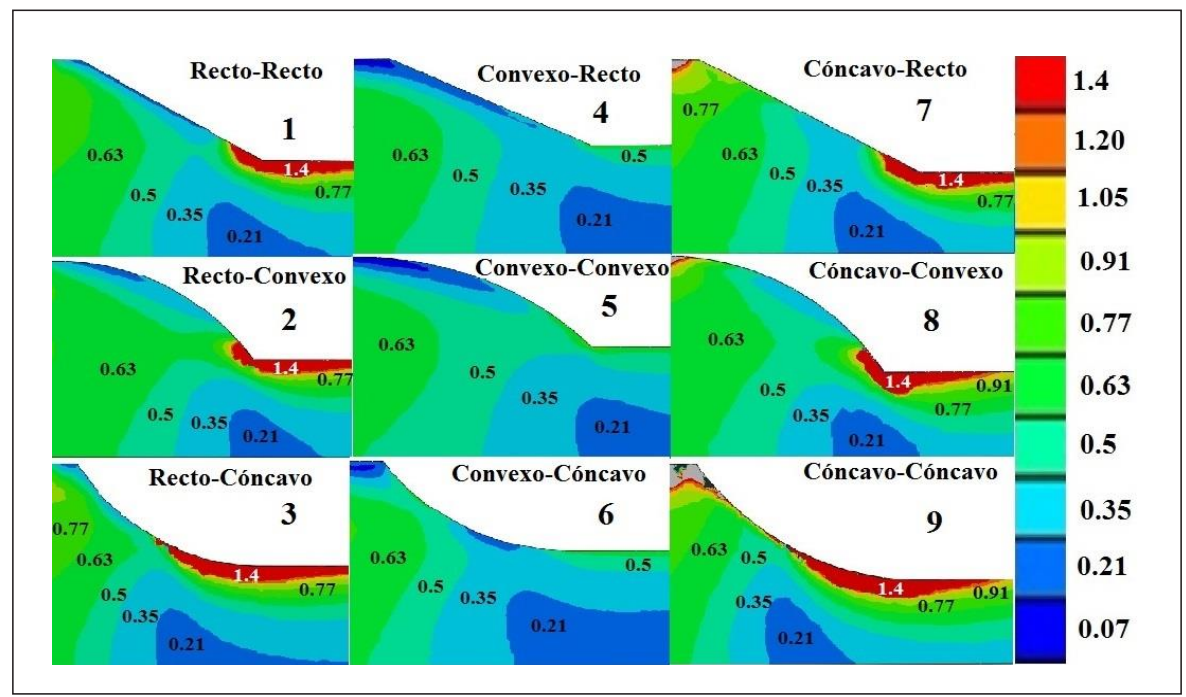

Figura 9. Contornos de indicadores potenciales de potenciales de falla en taludes con presencia agua.

Fuente: elaboración propia 
El indicador de susceptibilidad a la falla tiene la potencialidad de mostrar, en un único escalar, zonas dentro del talud que están más esforzadas y, consecuentemente tienen mayor posibilidad de inestabilidad.

Finalmente, es importante mencionar que los resultados encontrados en el presente trabajo no pueden ser usado en perfiles estratigráficos no homogéneos, conformados por materiales de diferentes propiedades físico-mecánicas o en ambientes geológicos en donde se encuentre presencia de planos pre-existentes en el terreno, que pueden constituir superficies potenciales de deslizamiento.

\section{CONCLUSIONES}

- Las geoformas convexas en perfil presentan una mayor susceptibilidad a la falla iniciando desde la pata del talud hacia la parte superior del mismo. Lo anterior indica que el mecanismo que se espera cuando se presenta una falla es del tipo progresivo

- Las geoformas cóncavas y rectas evidencian valores muy bajos de esfuerzos efectivos debido a la concentración del flujo en las zonas saturadas. Lo anterior se debe a la divergencia de las líneas de flujo desde el centro del talud

- El indicador de susceptibilidad a la falla tiene la potencialidad de mostrar en un único escalar zonas dentro del talud que están más esforzadas y consecuentemente tienen mayor posibilidad de inestabilidad

- El indicador de inestabilidad está en función de dos aspectos que condicionan la estabilidad de un talud bajo un modelo convencional de falla del tipo MohrCoulomb. Por un lado, a menores valores de presión media, más cerca se encuentra el material de la envolvente de falla. Por otro lado, a mayores esfuerzos de corte movilizados igualmente los puntos materiales se encuentran más cerca a la falla

- Los indicadores potenciales de falla permiten localizar zonas susceptibles a deslizamientos debido al efecto de la geoforma y al comportamiento divergente, convergente o uniforme del flujo

\section{AGRADECIMIENTOS}

Los autores agradecen a la Pontificia Universidad Javeriana por el apoyo económico para el desarrollo del presente trabajo bajo el proyecto de investigación No. 04756 "Retrospectiva de las catástrofes naturales en Colombia como insumo para la construcción de un sistema soporte de decisiones." 


\section{REFERENCIAS BIBLIOGRÁFICAS}

[1] R. Boroschek., y F. Domb, (2007). "Información para la Gestión de Riesgo de Desastres: Estudio de Caso de Cinco Países”. [En línea], acceso 24 de Enero, 2015, Disponible: http:// www.tesis.uchile.cl/tesis/uchile/2011/aq-acuna_d/pdfAmont/aq-acuna_d.pdf.

[2] M. Guillermo, et al. "Los suelos: estabilidad, productividad y degradación.” El medio ambiente en Colombia. Instituto de Hidrología, Meteorología y Estudios Ambientales, Bogotá, pp. 228-277, 2004.

[3] O. D. Cardona., et al. "Estudio sobre desastres ocurridos en Colombia: Estimación de pérdidas y cuantificación de costos." [En línea], acceso 26 de enero, 2015, Disponible http://www. desenredando. org/public/varios/2007/varios_omar/ERNDESASTRES_Colombia_LaRed. pdf Visitada el 24, 2004.

[4] C. Rodríguez. "Clase de estabilidad de taludes” Pontificia Universidad Javeriana, Bogotá, 2014.

[5] J. Akerman, "Fluvial aspects on slope processes and slope land-forms", Lond University, [En línea], acceso 11 de Febrero, 2015, Disponible http://www.nateko.lu.se/files/fluvial.pdf,2014.

[6] D. Wysocki y A. Schoeneberger "Geomorphology of soil landscapes." Handbook of soil science, 2000.

[7] E. Reid, Mark., y M. Richard Iverson. "Gravity-driven groundwater flow and slope failure potential: 2. Effects of slope morphology, material properties, and hydraulic heterogeneity." Water Resources Research, Vol 28.3, pp. 939-950, 1994.

[8] K. Hibbit, "Analysis of Geotechnical Problems with ABAQUS" [En línea] acceso 30 de enero, 2015, Disponible http://people.seas.harvard.edu/ viesca/articles/ABAQUSGeotechnicalProblemsv5.pdf,1996.

[9] P. Menetrey y K. Willam, “Triaxial failure criterion for concrete and its generalization" $A C I$ structural Journal 92, 1995.

[10] V. K. Puri, et al. "Geotechnical properties of crude oil contaminated sand."ASTM Special Technical Publication, pp. 1221 75-75, 1994.

[11] L. E. Bowles, "Foundation analysis and design”. 1988.

[12] V. Genuchten, M. T. "A closed-form equation for predicting the hydraulic conductivity of unsaturated soils." Soil science society of America journal 44.5, pp. 892-898, 1980.

[13] Y. Xu, y X. You, "Estimating parameters of van Genuchten model for soil water retention curve by intelligent algorithms." Appl. Math 7.5, pp. 1977-1983, 2013.

[14] D. S. Simulia, “ABAQUS 6.12 - Documentation” [En línea]. Disponible http://mat1.uibk.ac.at/ download/stix/Abaqus\%206.12\%20Documentation/, 2002. 